\section{Of Girths and Brains}

\author{
(D) M. Castillo, Editor-in-Chief
}

th is is now official: We Americans are no longer the heaviest in

the Western World. This ignominious claim belongs south of the border, to Mexico. The obesity rate of Mexicans $(32.8 \%)$ has now surpassed that of Americans (31.8\%). However, that is just obesity; if you take all of those who are overweight, the rate goes up to $70 \%$. Mexican child obesity has tripled in the last 10 years, and though a difficult concept to grasp, many obese individuals are also malnourished. Eating junk food makes one fatter but does not provide the necessary nutrients to be healthy. This weight gain trend is reflected throughout Latin America and other parts of the developing world. Lack of education and a desire to imitate the United States have led to this global problem, and now, being overweight far outstrips being underweight throughout the entire world (a landmark change in humanity that occurred in 2000). ${ }^{1}$ In Nauru and the Cook and Marshall Islands, obesity rates have reached $71 \%, 64 \%$, and $46 \%$, respectively, the highest rates worldwide. ${ }^{2}$ High ingestion of fried foods is probably the main culinary culprit, but Mexicans are also the highest consumers of sugary soft drinks in our hemisphere: 43 gallons (163 L) per person per year. Very soon, the world will have more than 900 million obese individuals. Obesity predominantly affects our 2 largest "minorities": Hispanics and African Americans; and despite what some reports say, their obesity rates have not changed significantly in the last few years. ${ }^{3}$ Because Hispanics and African Americans will soon together account for most of our population, it is very disturbing that by 2048, the entire US population will be obese if we do not stop this trend!

Apart from probably being unable to fit these patients into our MR imaging and CT units, neuroradiologists will become very involved in their care. Two of the most common obesity-related disorders are stroke and diabetes, with all of their neurologic complications. ${ }^{4}$ The current rate of stroke in the general population is about $0.5 \%$ per year, while in diabetics, it reaches $1.2 \%$ per year. In patients with diabetes, the risk of stroke increases with age, heart disease, previous stroke, smoking, and - not surprisinglywaist circumference. Of course, even without diabetes, stroke is more common in Hispanics and African Americans. Because the older population in the United States will increase from 13\% to $19 \%$ and Hispanics, from $14 \%$ to $19 \%$ by 2025 , stroke will become even more prevalent (the African American population will experience no significant growth and is expected to remain at the current $13 \%$ until about 2050). ${ }^{5}$

The cost of caring for patients with obesity is staggering. Currently, more than US $\$ 190$ billion is spent on it every year (21\% of all annual medical spending in the United States), and

http://dx.doi.org/10.3174/ajnr.A4156 even if obesity rates remained stable, that cost will increase to $\$ 550$ billion by 2030 . About $\$ 14$ billion is spent every year caring for obese children. Disease due to obesity costs businesses about $\$ 5$ billion in absenteeism every year. While Mexico is also a big country, its gross national product is only $13 \%$ of ours, so it faces a considerable challenge dealing with its obesity problem.

Although the main impetus for eating is a negative energy balance, many other nonhomeostatic factors, such as attractiveness of food, time and season (we tend to eat more during winter), and emotion (we eat more when sad or depressed), all affect our intake. An interesting observation is that these nonhomeostatic factors are so strong that they generally override the homeostatic ones. ${ }^{6}$ Different brain regions are involved for each of these mechanisms. The hypothalamus, parabrachial nucleus, ${ }^{*}$ and the nucleus of the tractus solitarius are all in charge of the homeostatic mechanisms. Various cortical locations, the amygdalae, ventral striata, hippocampi, and the substantia nigra, are in charge of the nonhomeostatic mechanisms and have complex connections with those in charge of homeostatic control. fMRI can identify activity in all of these regions. In one experiment, levels of peptide YY (PYY) in the bowel were manipulated and its effect was observed by fMRI. PYY activates vagal nerve afferents that activate homeostatic mechanisms and result in a reduction of food intake. fMRI is capable of showing that the homeostatic circuit is activated when the levels of PYY are manipulated. Another substance, leptin, a food-intake-reducing hormone, also results in brain changes measurable with fMRI. In a leptin-deficient state, individuals show activation of parts of the nonhomeostatic circuit and their desire for food increases, but when given leptin, the desire for food goes away, meaning the homeostatic mechanisms return.

There are 2 types of thin individuals: those who do not gain weight (obesity-resistant) and those who do (obesity-prone). ${ }^{6}$ Overfeeding significantly attenuates fMRI activation in the visual cortex of obesity-resistant individuals compared with obesity-prone ones, meaning that signals that operate to reduce food rewards and thus reduce intake in lean individuals are not present in those prone to gain weight. The practical applications of these observations are not certain, but manipulation of their function by medications or even brain stimulation/ablation could be possible in the future as a means of curing obesity.

Can obesity be inherited? Those who argue against this notion claim that altering our genes takes many generations and that the obesity epidemic is a fairly recent phenomenon; therefore, not enough time has gone by to change our genes. Those in favor state that the development of "energy-thrifty genes" occurred when

\footnotetext{
* The parabrachial nucleus complex (unknown to many neuroradiologists) is located at the junction of the midbrain and pons at the level of the superior cerebellar peduncle and is involved in the transmission of gustatory impulses.
} 
humans had less food, and now that we have an abundance of it, these genes favor the storage of fat and make us gain weight. Studies show that genetic factors may be responsible for $50 \%-80 \%$ of weight variations. ${ }^{7}$ About $5 \%$ of cases of obesity are monogenicthat is, caused by single gene defects ( 11 different genes identified so far). In polygenic obesity, more than 100 candidate genes have been identified. Genome-wide association studies have consistently pointed to the FTO gene as being a main culprit in obesity, and because it is a highly conserved gene, it is passed to subsequent generations. This gene leads to production of a protein that is predominantly expressed in the hypothalamus, so its absence may lead to the nonhomeostatic circuit overriding the homeostatic one. FTO gene polymorphism results in increased food intake in children and loss of control over eating, and FTO polymorphism carriers do not respond well to diets. What is more, FTO is also associated with diabetes independent of weight.

The second most common gene to be associated with obesity is called transmembrane protein 18. The polymorphism of this gene leads to weight gain and increased waist circumference and, even when adjusted for weight, also carries a higher risk of diabetes. Deletion of the $\mathrm{SH} 2 \mathrm{~B}$ adaptor protein (located in $16 \mathrm{p} 11.2$ ) leads to resistance to leptin and obesity and, once more, insulin resistance. Again, this gene is expressed in the hypothalamus and is capable of overriding homeostatic mechanisms. Neuronal growth regulator 1 (1p31.1) is also highly expressed in the brain, and when absent, weight gain, larger circumference, and diabetes ensue. Both of these latter genes also modulate the growth of adipose cells. The hypothalamus produces something called an agouti-related peptide, which is an antagonist of the melanocortin-4 receptor, which, if activated, decreases food intake. Homeostatic mechanisms in the hypothalamus block these receptors, increasing food intake, and if the gene that encodes for them is deficient and the receptors absent, satiety does not occur, leading to extreme eating, decline in energy use, and obesity.

Obese children may also have a genetic defect that makes them eat more carbohydrates. Animal studies show that mothers fed a long-standing high-fat diet produce offspring who demonstrate increased adiposity, glucose intolerance, and altered brain appetite regulators. ${ }^{8}$ Even in the face of only mild maternal overnutrition, these traits persist. It is hoped that knowledge of these gene defects will lead to personalized weight management and prediction of obesity and even perhaps gene manipulation in individuals at risk and that the effects of drugs may be monitored with fMRI. However, as always, things are not that easy, and epigenetic factors may further alter the functions of these genes. Ingestion of monounsaturated fats changes the way many of these genes act. So, do these genes change us or do we change these genes? Maybe both.

Once obesity is established, even in absence of diabetes, it increases arterial disease such as atherosclerosis. In one study, children with risk factors that included obesity had increased atherosclerosis progression in adulthood. ${ }^{9}$ Because $4 \%-6 \%$ of all US children are obese, the neurologic implications of these findings are important. Unfortunately, there is no easy fix because lifestyle modifications, behavior treatments, and even medications are only minimally effective and most participants remain obese after completion of these treatments. ${ }^{10}$ In obese children, the carotid arteries become thicker and stiff, and plasma markers of endothelial activation and injury are high. As the arteries stiffen, they cannot dilate to accommodate increased flow and the brain may not get enough blood when higher demand is in order.

Low back pain, the most common indication for lumbar spine MR imaging studies, is also correlated with weight. In one study in which participants were followed for 11 years, low back pain was either present at the beginning or developed during the study independent of other factors such as education, physical activity, and smoking. ${ }^{11}$ Neurosurgeons know that physical therapy and surgery commonly fail when the lumbar spine of obese patients is operated on. Infections and re-operation rates are also higher in the obese.

A newly recognized and significant risk factor for back pain in the obese is metabolic syndrome. Metabolic syndrome is associated with a special type of weight: a large waistline (individuals with excess fat in their abdomen but relatively little elsewhere). Other conditions associated with it are diabetes, high blood pressure, and high lipids. Overall, this syndrome is present in up to $20 \%$ of the adult US population and is highest in Hispanics. The prevalence of the syndrome is about $5 \%$ in those with a normal weight, nearly $60 \%$ in the obese, and nearly $39 \%$ in those with low back pain. ${ }^{12,13}$ Tumor necrosis factor- $\alpha(T N F-\alpha)$ is produced by individuals with the metabolic syndrome and is known to cause low back pain; when it is blocked, the pain disappears. Furthermore, aortic atherosclerosis associated with metabolic syndrome has been linked to degenerative disk disease and low back pain. The syndrome is also known to cause silent cerebral infarctions.

It is interesting that some investigators postulate that metabolic syndrome originates in the brain due to alterations in our circadian clocks. Normally, during sleep, our brain prepares our body for the next day's physical activity, but in modern life in which physical activity is minimal, this mechanism has been disrupted. The hypothalamus releases hormones and alters the function of the autonomic nervous system, resulting in changes in blood pressure, insulin, abdominal fat breakdown, and glucose uptake, but all of these activities are no longer needed as we sit at our desks all day long. The more abdominal fat we have, the greater the amount of adipokines we produce. Adipokines are cell-signaling proteins secreted by fatty tissues that have immunomodulating capacities, TNF- $\alpha$ being one of the most important ones. Additionally, adipose tissues produce hormones (called adipose-derived hormones), and their production becomes abnormal in patients with metabolic syndrome. One of these hormones is leptin, which as we saw above, can affect food intake. Obese individuals produce too much leptin, but instead of decreasing hunger, their brains become resistant to leptin and they just eat more.

There is a popular belief that up until the 1900 s, fat was seen as attractive, that in women it signified health and the ability to have babies, while in men, it meant prosperity. Newer research shows that most pre-Victorians and others before were thin, and their diets, nutritious. ${ }^{14}$ They ate many fruits and vegetables (mostly 
organic) and fiber, a diet akin to what we now call Mediterranean eating. No, they were not malnourished characters from a Dickens novel. They were actually healthy, and their physical activity is said to have been 3-4 times as much as ours. ${ }^{15}$ Recent evidence suggests that back then, life expectancy was not much different from now, the incidence of degenerative disease was $10 \%$ of ours, and cancer was basically nonexistent (of course, infections were rampant and childbirth fatalities and accidents were common). By the mid-Victorian times, diet and health had deteriorated significantly (cheap sugar, salted meats, and vegetable oils are just 3 popular products from the Agricultural Revolution responsible for obesity). The year 1900 was probably the last time we were a lean human race. Coming back to where I started, obesity was basically unknown in pre-Columbian Mesoamerica where the diet was gluten-free, low-carb, nutrient attenuated, and high in protein and fiber. Unfortunately, it is now in Mesoamerica where obesity is more prominent.

NB: For those who are interested in this topic, this is a very nice article: Caballero B. The global epidemic of obesity: an overview. Epidemiol Rev 2007;29:1-5

\section{REFERENCES}

1. Food and Agriculture Organization of the United Nations. The nutrition transition and obesity. http://www.fao.org/focus/e/obesity/ obes2.htm. Accessed January 22, 2014

2. LATINOVOICES. Mexico obesity rate surpasses the United States', making it fattest country in the Americas. http://www.huffingtonpost.com/ 2013/07/09/mexico-obesity_n_3567772.html. Accessed January 22, 2014

3. Flegal KM, Carroll MD, Kit BK, et al. Prevalence of obesity and trends in the distribution of body mass index among US adults, 1999-2010. JAMA 2012;307:491-97

4. Centers for Disease Control and Prevention. Adult obesity facts. http://www.cdc.gov/obesity/data/adult.html. Accessed January 22, 2014

5. Passel JS, Cohn D. US population projections: 2005-50. Pew Research Hispanic Trends Project. February 11, 2008. http://www. pewhispanic.org/2008/02/11/us-population-projections-2005-2050. Accessed January 28, 2014

6. Grill HJ, Skibicka KP, Hayes MR. Imaging obesity: fMRI, food reward, and feeding. Cell Metabolism 2007;6:423-25

7. Moleres A, Martinez JA, Marti A. Genetics of obesity. Curr Obes Rep 2013;2:23-31

8. Rajia S, Chen H, Morris MJ. Maternal overnutrition impacts offspring adiposity and brain appetite markers: modulation by postweaning diet. J Neuroendocrinol 2010;22:905-14

9. Juonala M, Viikari JSA, Kanonen M, et al. Life-time risk factors and progression of carotid atherosclerosis in young adults: the cardiovascular risk in young Finns study. Eur Heart J 2010;31:1745-51

10. Kelly A, Barlow SE, Rao G, et al. Severe obesity in children and adolescents: identification of health risks, and treatment approaches - a scientific statement from the American Heart Association. Circulation 2013;128:1698-712

11. Heuch I, Heuch I, Hagen K, et al. Body mass index as a risk factor for developing chronic low back pain: a follow-up in the Nord-Trondelag health study. Spine 2013;38:133-39

12. Park YW, Palaniappan L Heshka S, et al. The metabolic syndrome: prevalence and associated risk factor findings in the US population from the third national health and nutrition examination survey, 1988-1994. Arch Intern Med 2003;163:427-36

13. Ha JY. Evaluation of metabolic syndrome in patients with chronic low back pain: using the fourth Korea national health and nutrition examination survey data. Chonnam Med J 2011;47:160-64
14. Westcott P. Healthy eating Victorian style. Saga. http://www. saga.co.uk/health/healthy-Eating/healthy-eating-victorian-style. aspx. Accessed January 30, 2014

15. Clayton P, Rowbotham J. How the mid-Victorians worked, ate and died. Int J Environ Res Public Health 2009;6:1235-53

\section{EDITORIAL - -}

\section{Viewpoints on the ARUBA Trial}

\author{
J.P. Mohr, A. Hartmann, H. Kim, J. Pile-Spellman, and C. Stapf
}

A Randomized Trial of Unruptured Brain Arteriovenous Malformations (ARUBA), the first randomized clinical trial for brain arteriovenous malformations (bAVMs), was planned as a straightforward simple attempt to learn whether deferring intervention for a bAVM that had not bled would prove superior to incurring the risks of intervention needed to eradicate the lesion. The trial was justified by longitudinal data on true natural history (ie, for those receiving no intervention to eradicate the bAVM), reports of mild syndromes from many who had bled, and literature with treatment outcomes that were a mix of those who had bled before treatment versus those who had not. Having no wish to disturb current established interventional practice, the investigators offered randomization only to those whose bAVMs were considered suitable for eradication; none whose bAVMs were deemed too daunting for intervention would be eligible. Medical management for headaches and seizures is well-established, but no standards have yet appeared dictating interventional management. Widely misquoted literature citing annual hemorrhage rates approximating $4 \%$ and estimates of low risks for intervention allowed the assumption that the trial might well end within 5 years with a win for intervention. ${ }^{1}$ Moreover, more insight would be gained for the true natural history.

The National Institute of Neurological Disorders and Stroke (NINDS) application followed well-established guidelines: an aim, a primary null hypothesis, clear and simple primary outcomes, and a host of secondary aims should enough data be available for useful analysis, with all information posted on the Web. ${ }^{2}$ Participating centers sought, were offered, and were assumed to use their experience-based choices of interventions to achieve the goals of lesion eradication. The 39 active centers randomized fully $61 \%$ of those eligible. They also showed their qualifications by publishing fully 630 PubMed references for bAVMs during 20002010. Outcomes were reported at fixed intervals and after each intervention (many interventions not yielding single-stage eradication) and were adjudicated by a distinguished 4-member panel. An NINDS-appointed equally distinguished Data and Safety Monitoring Board (DSMB) provided independent oversight of study conduct and participant safety. National Institutes of Health (NIH) trials are typically funded in cycles of 5 years or less. Continuation depends on successful review and priority scores

- Indicates open access to non-subscribers at www.ajnr.org

http://dx.doi.org/10.3174/ajnr.A4204

AJNR Am J Neuroradiol 36:613-22 Apr 2015 www.ajnr.org 615 